\section{Exposição ocupacional ao ruído e acidentes do trabalho}

\author{
Occupational noise exposure \\ and work accidents
}

I Faculdade de Ciências
Médicas, Universidade
Estadual de Campinas,
Campinas, Brasil.
2 Faculdade de Medicina
de Botucatu, Universidade
Estadual Paulista,
Botucatu, Brasil.
3 Departamento
de Fonoaudiologia,
Universidade Metodista
de Piracicaba,
Piracicaba, Brasil.
Correspondência
A. Dias
Departamento de Medicina
Preventiva e Social,
Faculdade de Ciências
Médicas, Universidade
Estadual de Campinas.
Rua Jorge Barbosa de Barros
526, Botucatu, SP
18610-304, Brasil.
adias@fmb.unesp.br

Abstract

The purpose of this study was to verify whether occupational noise exposure is a significant risk factor for work accidents in the city of Piracicaba, São Paulo State, Brazil. This hospital-based case-control study included 600 workers aged 15-60 who suffered typical occupational accidents between May and October 2004 and were seen at the Piracicaba Orthopedics and Trauma Center. The control group comprised 822 workers, aged 15-60, who were also seen at the Center, and either had a non-occupational accident or were accompanying someone who had suffered an accident. A multiple logistic regression model was adjusted with work accident as an independent variable, controlled by covariables of interest such as noise exposure. The risk of having a work accident was about twice as high among workers exposed to noise, after controlling for several covariables. Occupational noise exposure not only affected auditory health status but was also a risk factor for work accidents.

Occupational Noise; Occupational Accidents; Occupational Risks
Adriano Dias 1,2

Ricardo Cordeiro 1

Cláudia Giglio de Oliveira Gonçalves 3

\section{Introdução}

Os acidentes do trabalho são o maior agravo à saúde dos trabalhadores. Constituem um importante problema de saúde pública, tanto em países em desenvolvimento, quanto em países desenvolvidos. No Brasil, são oficialmente definidos como "aqueles que ocorrem pelo exercício do trabalho a serviço da empresa, ou ainda pelo exercício do trabalho dos segurados especiais, provocando lesão corporal ou perturbação funcional que cause morte, perda ou redução, permanente ou temporária, da capacidade de trabalho" 1. Podem ser classificados como: acidente-tipo, que é aquele que ocorre a serviço da empresa ou acidente de trajeto, que é o ocorrido no momento em que o trabalhador se desloca para o local de trabalho ou nos horários das refeições 2 . Diferentemente do que o nome sugere, os acidentes de trabalho não são eventos fortuitos ou acidentais 3,4 , mas fenômenos socialmente determinados e preveníveis 4,5 .

A literatura especializada internacional aponta que trabalhadores expostos ao ruído ocupacional intenso apresentam risco aumentado de se acidentarem quando comparados a trabalhadores não expostos 6,7. Refere também que a implantação de Programas de Conservação Auditiva (PCA) abrangendo trabalhadores expostos ao ruído ocupacional, tendo em vista o objetivo de prevenção da exposição e do dano auditivo, diminui consideravelmente o risco de acidentes 8,9. 
O objetivo desta pesquisa foi verificar se a exposição ocupacional ao ruído constitui um risco importante para acidentes do trabalho em cidade industrializada do interior do Estado de São Paulo, Brasil.

\section{Método}

Este estudo fez parte de uma investigação maior, objetivando aprimorar as ações de vigilância em saúde do trabalhador no Município de Piracicaba, São Paulo, Brasil, denominada DIATEP: Diagnóstico e Controle de Acidentes do Trabalho em Piracicaba 10. Como parte de seu desenvolvimento, realizou-se um estudo caso-controle de base hospitalar, tendo como população fonte os trabalhadores de Piracicaba, investigando a possibilidade de a exposição ocupacional ao ruído ser um fator de risco para acidentes do trabalho.

Foram critérios para inclusão de casos: ser morador da cidade de Piracicaba, ter idade entre 15 e 60 anos, ser atendido no pronto-socorro do Centro de Ortopedia e Traumatologia de Piracicaba (COT) em decorrência de acidente do trabalho típico e concordar em participar do estudo por meio de Termo de Consentimento Livre e Esclarecido. Para a inclusão dos controles, os critérios foram: ser morador da cidade de Piracicaba, ter idade entre 15 e 60 anos, ser atendido no COT por qualquer motivo diferente de acidente do trabalho ou ser acompanhante de paciente atendido e concordar em participar do estudo por meio de Termo de Consentimento Livre e Esclarecido.

Após a apresentação dos objetivos do estudo e a obtenção por escrito do consentimento em dele participar, foram colhidas as informações de casos e controles por entrevistadores treinados que aplicavam questionário indagando sobre diversas variáveis ocupacionais e não ocupacionais. Dentre outras, o questionário aplicado continha as seguintes questões: (a) sexo - variável categórica que informa o gênero dos casos e controles; (b) idade - variável contínua (anos); (c) anos de escolaridade - variável numérica que informa o número de anos completos de escolaridade; (d) tipo de trabalho variável categórica dicotômica que informa se o trabalhador trabalhava com ou sem contrato legal de trabalho; (e) tipo de turno de trabalho variável categórica que informa o tipo de turno de trabalho, categorizada como "turno diurno fixo" (escolhida como nível basal), "turno noturno fixo" e "turno alternado"; (f) jornada diária média de trabalho - variável numérica (horas); (g) média de horas extras de trabalho semanais - variável numérica (horas).
Nas entrevistas, foram colhidas descrições detalhadas das atividades laborais atuais. Com base nessas informações, as ocupações exercidas por casos e controles foram classificadas segundo os nove grandes grupos da Classificação Brasileira de Ocupações 11: "cientistas” (nível basal), "policiais”, "gerentes”, “técnicos”, "administrativos", "serviços”, "agricultores”, “operários” e "manutenção".

A investigação sobre exposição a ruído e algumas queixas auditivas importantes se deu por meio das questões abaixo:

- "Como é normalmente a intensidade do ruído em seu local de trabalho?" - que admitia quatro respostas: "não há ruído" (nível basal), "fraco", "médio" e "forte";

- "Como é normalmente a duração do ruído em seu local de trabalho?" - que admitia três respostas: "não há ruído" (nível basal), "continuamente durante a jornada" e "intermitente durante a jornada";

- "No momento em que você sofreu o acidente [casos] ou no último dia em que você trabalhou [controles] como estava o ruído?" - que admitia cinco respostas: "não havia ruído" (nível basal), "fraco", "médio", "forte" e "não lembro";

- “Você sente zumbido?" - que admitia três respostas: "nunca" (nível basal), "sempre" e "às vezes";

- “Você sente tontura?" - que admitia três respostas: "nunca” (nível basal), "sempre” e "às vezes";

- "Você tem dificuldade de audição?" - que admitia duas respostas: "sim" e "não";

- "O ruído no trabalho lhe causa incômodo?" que admitia duas respostas: "sim" e "não".

As respostas às questões que envolviam variáveis categóricas com mais de dois níveis foram tratadas como variáveis dummy, conforme o nível basal informado.

$\mathrm{Na}$ análise, inicialmente, foram ajustados modelos univariados de regressão logística simples 12 , tendo como variável resposta dicotômica à ocorrência de acidente (controle $=0$, caso $=$ 1) e como variável preditora cada uma das variáveis acima referidas.

Posteriormente, foi ajustado um modelo de regressão logística múltipla 12 , em que a variável resposta categórica dicotômica foi a ocorrência de acidente (controle $=0$, caso $=1$ ) e as variáveis preditoras foram aquelas que nos modelos univariados produziram estimativas de odds ratio (OR) com valor $\mathrm{p} \leq 0,2513$.

Este estudo recebeu parecer favorável do Comitê de Ética em Pesquisa da Faculdade de Medicina de Botucatu, Universidade Estadual Paulista. 


\section{Resultados}

Os dados foram coletados em todos os dias úteis compreendidos entre 16 de maio e 29 de outubro de 2004, compreendendo 600 casos e 822 controles.

A Tabela 1 apresenta a distribuição por idade do total de acidentados típicos selecionados para o estudo. Salienta-se que mais 53\% desses acidentes atingiram adultos jovens com até trinta anos, tendo $12 \%$ dos acidentados até vinte anos de idade.

A maioria dos acidentes consistiu de contusões $(46,82 \%)$, seguida por entorses $(14,88 \%)$, ferimentos corto-contusos $(10,36 \%)$ e fraturas (9,03\%), que atingiram, principalmente, as mãos $(36,63 \%)$, os pés $(18,39 \%)$, os membros superiores $(14,71 \%)$, os membros inferiores $(14,04 \%)$ e a coluna $(9,36 \%)$. As causas imediatas dos acidentes identificados foram, em sua maioria, aquelas relacionadas às máquinas e equipamentos $(23,74 \%)$, quedas de objetos $(23,57 \%)$, esforço excessivo ou excesso de peso $(13,04 \%)$ e aquelas ocasionados por quedas $(8,53 \%)$.

A Tabela 2 apresenta estatísticas obtidas nos ajustes univariados acima mencionado.

Aplicando-se o modelo logístico multivariado referido, contendo apenas as variáveis que obtiveram valor $\mathrm{p}<0,25$ nos modelos univariados, observou-se que as variáveis "trabalhador atribui ruído médio no trabalho" e "trabalhador atribui ruído forte no trabalho" mostraram-se fatores de risco para acidentes do trabalho, com estimativas ajustadas de OR iguais a 1,371 ( $\mathrm{p}=$ 0,0113; IC95\%: 1,074-1,751) e 1,902 ( $\mathrm{p}=0,0003$; IC95\%: 1,339-2,703), respectivamente. A Tabela 3 apresenta estatísticas obtidas no ajuste do modelo logístico incluindo todas as variáveis que nele permaneceram. As estimativas dos coeficientes para as outras variáveis testadas no modelo produziram resultados com valor $\mathrm{p}$ maiores que 0,05 e foram descartadas no ajuste.

Não se observou nenhum termo de interação estatisticamente significativo $(\alpha=0,05)$ entre as variáveis selecionadas. Analisando-se os resíduos do ajuste, não se verificaram violações dos pressupostos do modelo logístico utilizado. Todas as análises foram feitas utilizando-se o programa SAS versão 8.2 (SAS Institute, Cary, Estados Unidos).

\section{Discussão}

No ajuste multivariado, conforme mostra a Tabela 3 , as variáveis "trabalhador atribui ruído médio no trabalho" e "trabalhador atribui ruído forte no trabalho" foram identificadas como
Tabela 1

Distribuição dos acidentados segundo a idade, em estudo caso-controle. Piracicaba, São Paulo, Brasil, 2004.

\begin{tabular}{lrr}
\hline Idade (anos) & Freqüência $(\mathbf{n})$ & $\%$ \\
\hline $11-20$ & 72 & 12,00 \\
$21-30$ & 249 & 41,50 \\
$31-40$ & 132 & 22,00 \\
$41-50$ & 113 & 18,83 \\
$51-60$ & 34 & 5,67 \\
Total & 600 & 100,00 \\
\hline
\end{tabular}

Tabela 2

Estatísticas obtidas nos ajustes logísticos univariados em estudo caso-controle. Piracicaba, São Paulo, Brasil, 2004

\begin{tabular}{|c|c|c|}
\hline Variável & OR & Valor $p$ \\
\hline Sexo & 1,892 & $<0,0001$ \\
\hline Idade & 1,001 & 0,7823 \\
\hline Anos de escolaridade & 0,897 & $<0,0001$ \\
\hline Trabalho sem contrato legal & 0,766 & 0,0612 \\
\hline Turno alternado & 1,611 & 0,1541 \\
\hline Turno noturno & 0,518 & 0,0393 \\
\hline Jornada diária & 1,072 & 0,0425 \\
\hline Horas extras semanais & 1,019 & 0,0119 \\
\hline Administrativos & 1,755 & 0,0786 \\
\hline Agricultores & 1,783 & 0,2811 \\
\hline Manutenção & 6,310 & $<0,0001$ \\
\hline Operários & 4,233 & $<0,0001$ \\
\hline Serviços & 2,492 & 0,0002 \\
\hline Trabalhador atribui ruído fraco no trabalho & 1,442 & 0,0222 \\
\hline Trabalhador atribui ruído médio no trabalho & 1,859 & $<0,0001$ \\
\hline Trabalhador atribui ruído forte no trabalho & 2,929 & $<0,0001$ \\
\hline Ruído contínuo & 2,271 & $<0,0001$ \\
\hline Ruído intermitente & 1,620 & $<0,0013$ \\
\hline $\begin{array}{l}\text { Ruído fraco no momento do acidente (casos) } \\
\text { ou na última jornada de trabalho (controles) }\end{array}$ & 0,437 & $<0,0001$ \\
\hline $\begin{array}{l}\text { Ruído médio no momento do acidente (casos) } \\
\text { ou na última jornada de trabalho (controles) }\end{array}$ & 0,552 & $<0,0001$ \\
\hline $\begin{array}{l}\text { Ruído forte no momento do acidente (casos) } \\
\text { ou na última jornada de trabalho (controles) }\end{array}$ & 0,965 & 0,8502 \\
\hline Sempre tem zumbido & 1,009 & 0,9675 \\
\hline Às vezes tem zumbido & 0,948 & 0,7481 \\
\hline Sempre tem tontura & 0,733 & 0,2109 \\
\hline Às vezes tem tontura & 0,786 & 0,1260 \\
\hline Dificuldade de audição & 1,148 & 0,4073 \\
\hline Ruído sempre incomoda & 1,016 & 0,9161 \\
\hline Ruído às vezes incomoda & 1,071 & 0,7197 \\
\hline
\end{tabular}


Estatísticas obtidas no ajuste logístico multivariado em estudo caso-controle. Piracicaba, São Paulo, Brasil, 2004.

\begin{tabular}{lccc}
\hline Variável & $\begin{array}{c}\text { Estimativa do } \\
\text { parâmetro } \beta\end{array}$ & Valor $\mathbf{p}$ & $\begin{array}{c}\text { Estimativa de } \\
\text { OR (IC95\%) }\end{array}$ \\
\hline Trabalhador atribui ruído médio no trabalho & 0,3158 & 0,0113 & $1,371(1,074-1,751)$ \\
Trabalhador atribui ruído forte no trabalho & 0,6431 & 0,0003 & $1,902(1,339-2,703)$ \\
Manutenção & 0,7659 & 0,0031 & $2,151(1,294-3,575)$ \\
Operários & 0,8430 & $<0,0001$ & $2,323(1,630-3,311)$ \\
Serviços & 0,4484 & 0,0226 & $1,566(1,065-2,302)$ \\
Escolaridade & $-0,0814$ & $<0,0001$ & $0,922(0,889-0,956)$ \\
\hline
\end{tabular}

$\chi^{2}$ Razão de verossimilhança $=92,1435$, g.l. $=6 ; p=0,0001$.

riscos para acidentes do trabalho, com estimativas ajustadas de OR iguais a $1,371(\mathrm{p}=0,0113$; IC95\%: 1,074-1,751) e 1,902 ( $\mathrm{p}=0,0003$; IC95\%: 1,339-2,703), respectivamente, controladas para aquelas variáveis mostradas na Tabela 2 . Nota-se que a experiência e subjetividade do trabalhador são importantes e valorizá-las possibilita a sistematização de um saber que auxiliará no processo de investigação da saúde dos trabalhadores, como aquele gerado, por exemplo, pelo modelo operário italiano 14 . Facchini et al. 15, aplicando tal modelo para a identificação dos riscos e danos do ambiente de trabalho na saúde dos trabalhadores, constataram que os dados obtidos, quando comparados por vistorias técnicas realizadas numa indústria química, comprovaram as observações realizadas pelos trabalhadores.

A probabilidade de ocorrência de um acidente do trabalho não é distribuída homogeneamente entre diferentes trabalhadores executando diferentes tarefas em diferentes ocupações. Tarefas e ocupações diferenciam-se quanto ao grau de exposição aos riscos. Ambientes de trabalho ruidosos podem conter (e geralmente contêm) outros riscos ocupacionais para acidentes que não o ruído propriamente dito. Por isso, na análise logística múltipla efetuada, as estimativas de risco obtidas foram controladas, entre outras variáveis, para escolaridade e grandes grupos de ocupação. Esta foi uma estratégia para controlar o possível confundimento decorrente da falta de comparabilidade entre casos e controles quanto às ocupações. De fato, as estimativas ajustadas de risco associadas às variáveis "trabalhador atribui ruído médio no trabalho" e "trabalhador atribui ruído forte no trabalho", mostradas na Tabela 3, foram diferentes das estimativas brutas, apresentadas na Tabela 2 , sugerindo a existência de confundimento. Ainda com relação ao mostrado pela Tabela 2 e aos possíveis confundimentos, observa-se que, nas análises univariadas, figurava entre os fatores de risco para acidentes do trabalho a variável "trabalhador atribui ruído fraco no trabalho", que não permaneceu no modelo multivariado ajustado.

No Brasil, Barreto et al. 16, em estudo casocontrole aninhado a uma coorte de trabalhadores metalúrgicos acompanhada entre 1977 e 1990, encontrou associação significativa entre exposição ao ruído industrial e ocorrência de acidentes do trabalho fatais, ajustada para vários fatores de confusão. Também em nosso meio, Cordeiro et al. 7, em estudo caso-controle de base populacional realizado em 2004, encontraram uma associação entre exposição moderada a ruído ocupacional e acidente do trabalho estimada em 3,7 (IC95\%: 1,8-7,4), bem como uma associação entre exposição intensa a ruído ocupacional e acidente do trabalho estimada em 5,0 (IC95\%: 2,8-8,7).

Em artigo de revisão, Kjellberg 17 refere que altos níveis de ruído no local de trabalho estão associados a altas taxas de acidentes do trabalho.

Em um estudo caso-controle realizado entre trabalhadores de um estaleiro na Holanda, entre 1986 e 1987, Moll van Charante \& Mulder 18 encontraram associação entre exposição ao ruído industrial maior que $82 \mathrm{~dB}$ e ocorrência de acidentes do trabalho, estimando como 1,8 (IC95\%: 1,2-2,9) a OR dessa associação, ajustado para vários fatores de confusão. Estes autores notaram que essa associação não se fazia presente entre os trabalhadores com perda auditiva já instalada.

Em 1992, Melamed et al. 19 encontraram, em um estudo transversal realizado entre 2.368 trabalhadores industriais, associação entre exposição ao ruído maior que $85 \mathrm{~dB}$ e acidentes do trabalho. Novamente, em 2004, Melamed et al. 20 voltaram a demonstrar a associação entre 
esses fatores, na segunda fase do mesmo estudo, agora longitudinal, buscando as estimativas de risco do acidente do trabalho associadas à exposição ao ruído ocupacional e às interações entre diversos fatores.

Berger et al. 8 referem que trabalhadores sem proteção auditiva atuando em ambientes ruidosos têm maior chance de acidentarem-se. Lindberg et al. 21, em estudo com dois mil trabalhadores suecos que identificou cerca de 250 acidentes do trabalho durante dez anos, mostram que a exposição ao ruído está associada aos acidentes do trabalho, com OR ajustado de 1,1 (IC95\%: 1,02-1,1) para cada ano de exposição.

Os valores das estimativas de OR obtidas neste estudo encontram-se em consonância com as referências da literatura internacional. Chama a atenção a consistência da associação entre exposição ocupacional a ruído e acidente do trabalho, acima apontada. Aprofundando a investigação da natureza dessa associação, é interessante observar que o ruído ocupacional impõe ao trabalhador dificuldades de comuni-

\section{Resumo}

O objetivo deste estudo foi verificar se a exposição ocupacional ao ruído é um fator de risco relevante para acidentes do trabalho na cidade de Piracicaba, São Paulo, Brasil. Foi realizado um estudo caso-controle de base hospitalar. Os casos foram 600 trabalhadores entre 15 e 60 anos que sofreram acidentes ocupacionais típicos entre maio e outubro de 2004, atendidos na Central de Ortopedia e Traumatologia de Piracicaba. Os controles foram 822 trabalhadores entre 15 e 60 anos que sofreram acidente não ocupacional e receberam o primeiro atendimento no serviço ou foram trabalhadores acompanhantes dos acidentados. Foi ajustado um modelo de regressão logística múltipla, tendo como variável independente o acidente do trabalho, controlada por co-variáveis de interesse, entre elas a exposição ao ruído. Estimou-se que o risco de sofrer acidente do trabalho é cerca de duas vezes maior entre trabalhadores expostos ao ruído, controlado para diversas co-variáveis. A exposição ocupacional ao ruído não só deteriora a saúde auditiva do trabalhador, mas também se constitui em fator de risco para acidentes do trabalho.

Ruído Ocupacional; Acidentes de Trabalho; Riscos Ocupacionais

\section{Colaboradores}

A. Dias e R. Cordeiro participaram do planejamento global do estudo, coleta e análise global dos dados e redação do texto. C. G. O. Gonçalves colaborou na análise das informações sobre ruído. cação (na detecção, discriminação, localização e identificação das fontes sonoras, assim como na inteligibilidade de fala) 8,22,23,24, de manutenção da atenção e concentração 25,26 , de memória 25,27 , além do estresse 19,28,29,30 e fadiga excessiva 26,28. Estes são fatores sabidamente envolvidos na gênese de acidentes do trabalho 31 . Conseqüentemente, parece haver relação causal entre ruído ocupacional e acidente do trabalho, o que remete de imediato para o campo da prevenção dos acidentes de trabalho.

Dado que a magnitude da exposição ocupacional ao ruído foi estimada em 600 milhões de trabalhadores no mundo em 1998 32, e que, por exemplo, apenas nos Estados Unidos ocorrem anualmente mais de cinco milhões de acidentes do trabalho 33 , os achados aqui apresentados justificam o investimento em programas de conservação auditiva particularmente voltados para o controle da emissão de ruídos na fonte, objetivando não apenas a manutenção da saúde auditiva, mas também a diminuição da acidentabilidade dos trabalhadores.

\section{Agradecimentos}

Trabalho realizado no Centro de Referência em Saúde do Trabalhador de Piracicaba e financiado pela Fundação de Amparo à Pesquisa do Estado de São Paulo (00/13719-3).

\section{Referências}

1. Decreto n. 2.172. Aprova o regulamento dos benefícios da Previdência Social. Diário Oficial da União 1999; 12 mai. http://www.previdencia.gov. br/pg_secundarias/paginas_perfis/perfil_Empre gador_10_04-A5.asp (acessado em 08/Dez/ 2005).

2. Waldvogel BC. A população trabalhadora paulista e os acidentes do trabalho fatais. São Paulo Perspect $2003 ; 17: 42-53$.

3. Tsai SP, Bernacki EJ, Dowd CM. The relationship between work-related and non-work-related injuries. J Community Health 1991; 16:205-12.

4. Vilela RAG. Acidentes do trabalho com máquinas: identificação de riscos e prevenção. São Paulo: Central Única dos Trabalhadores; 2000.

5. Dwyer T. Life and death at work: industrial accidents as a case of socially produced error. New York: Plenum Press; 1991.

6. Cohen A. Industrial noise and medical absence and accident record data on exposed workers. In: Proceedings of the International Congress on Noise as a Public Health Problem. Washington DC: US Environmental Protection Agency; 1976. p. 441-53.

7. Cordeiro R, Clemente AP, Diniz CS, Dias A. Occupational noise as a risk factor for work-related injuries. Rev Saúde Pública 2005; 39:461-6. 
8. Berger EH, Royster LH, Royster JD, Driscoll DP, Layne M. The noise manual. $5^{\text {th }}$ Ed. Akron: American Industrial Hygiene Association; 2000.

9. Cohen A. The influence of a company hearing conservation programme on extra-auditory problems in workers. J Safety Res 1976; 8:146-62.

10. Cordeiro R, Vilela RAG, Medeiros MAT, Gonçalves CGO, Bragantini CA, Varolla AJ, et al. O sistema de vigilância de acidentes do trabalho de Piracicaba, São Paulo, Brasil. Cad Saúde Pública 2005; 21: 1574-83.

11. Instituto Brasileiro de Geografia e Estatística. Classificação de ocupações - censo demográfico de 2000. http://www.ibge.gov.br/concla/ocupacao/ cbo/cbo.shtm (acessado em 01/Jul/2002).

12. Hosmer Jr. D, Lemeshow S. Applied logistic regression. New York: John Wiley \& Sons; 2000.

13. Rothman KJ, Greenland S. Modern epidemiology. Philadelphia: Lippincott-Raven; 1998.

14. Laurell AC. Ciencia y experiencia obrera. Cuadernos Políticos 1984; 41:63-83.

15. Facchini LA, Welderpass E, Tomasi E. Modelo operário e percepção de riscos ocupacionais e ambientais: o uso exemplar de estudo descritivo. Rev Saúde Pública 1991; 25:394-400.

16. Barreto SM, Swerdlow AJ, Smith PG, Higgins CD A nested case-control study of fatal work related injuries among Brazilian steel workers. Occup Environ Med 1997; 54:599-604.

17. Kjellberg A. Subjective, behavioral and psychophysiological effects of noise. Scand J Work Environ Health 1990; 16 Suppl 1:29-38.

18. Moll van Charante AW, Mulder PG. Perceptual acuity and the risk of industrial accidents. Am J Epidemiol 1990; 131:652-63.

19. Melamed S, Luz J, Green MS. Noise exposure, noise annoyance and their relation to psychological distress, accident and sickness absence among blue-collar workers - the Cordis Study. Isr J Med Sci 1992; 28:629-35.

20. Melamed S, Fried Y, Froom P. The joint effect of noise exposure and job complexity on distress and injury risk among men and women: the cardiovascular occupational risk factors determination in Israel study. J Occup Environ Med 2004; 46:1023-32.

21. Lindberg E, Carter N, Gislason T, Janson C. Role of snoring and daytime sleepiness in occupational accidents. Am J Respir Crit Care Med 2001; 164:2031-5.
22. Hétu R, Quoc HT. Psychoacoustic performance in workers with NIHL. In: Axelson A, Bordigrevink H, Hamernik RP, Hellstrom P, Henderson D, Salvi RJ, editors. Scientific basis of noise-induced hearing loss. New York: Thieme; 1996. p. 264-85.

23. Shimizu T, Makishima K, Yoshida M, Yamagishi H. Effect of background noise on perception of English speech for Japanese listeners. Auris Nasus Larynx 2002; 29:121-5.

24. Berglund B, Lindvall T, Schwela DH. Guidelines for commuinity noise. Geneva: World Health Organization; 1999.

25. Berglund B, Lindvall T. Community noise. Stockholm: World Health Organization; 1995.

26. Ribeiro HP, Lacaz FAC. Acidentes de trabalho. In: Departamento Intersindical de Estudos e Pesquisas de Saúde e dos Ambientes de Trabalho, organizador. De que adoecem e morrem os trabalhadores. São Paulo: Departamento Intersindical de Estudos e Pesquisas de Saúde e dos Ambientes de Trabalho; 1984. p. 65-85.

27. World Health Organization. Occupational and community noise. http://www.who.int/mediacentre/factsheets/fs258/en/ (acessado em 20/ Fev/2002).

28. Ferreira Jr. M. Perda auditiva induzida pelo ruído. In: Ferreira Jr. M, organizador. Saúde no trabalho. São Paulo: Editora Roca; 2000. p. 262-85.

29. Gessinger R, Castoldi L, Fensterseifer LM. Efeitos psicossociais da perda auditiva induzida pelo ruído (PAIR). In: Nudelmann AA, Costa EA, Seligman J, Ibanez NR, organizadores. PAIR: perda auditiva induzida pelo ruído. Porto Alegre: Editora Bagaggem; 1997. p. 251-4.

30. Santos UP. Exposição a ruído: avaliação de riscos, danos à saúde e prevenção. In: Santos UP, organizador. Ruído: riscos e prevenção. São Paulo: Editora Hucitec; 1999. p. 3-6.

31. Swaen GM, van Amelsvoort LG, Bultmann U, Kant IJ. Fatigue as a risk factor for being injured in an occupational accident: results from the Maastricht Cohort Study. Occup Environ Med 2003; 60 Suppl 1:i88-92.

32. Alberti PW. Noise, the most ubiquitous pollutant. Noise Health 1998; 1:3-5.

33. Smith GS, Wellman HM, Sorock GS, Warner M, Courtney TK, Pransky GS, et al. Injuries at work in the US adult population: contributions to the total injury burden. Am J Public Health 2005; 95:1213-9.

Recebido em 04/Out/2005

Versão final reapresentada em 14/Dez/2005 Aprovado em 17/Jan/2006 